\title{
Can a Stent Retriever Damage the JET 7 Reperfusion Catheter?
}

\author{
(D). Pearly Ti, (D) L. Yeo, and (D) G. Anil
}

\section{ABSTRACT}

SUMMARY: We encountered 2 separate instances of damage to JET 7 reperfusion catheters when they were used in conjunction with a stent retriever during mechanical thrombectomy. On both occasions, after 1 or 2 passes with a stent retriever, we found that the distal end of the catheter was frayed and it ballooned up on flushing with saline. This mechanical failure could potentially lead to serious complications; hence, it should be shared with fellow neurointerventionalists.

ABBREVIATION: DAC $=$ distal access catheters

$I^{\mathrm{n}}$ endovascular treatment for acute ischemic stroke with largevessel occlusion, stent retriever thrombectomy has been the default standard of care. ${ }^{1}$ The Cost and Medical Care of Patients With Advanced Serious Illness in Singapore trial proved that aspiration with large-bore distal access catheters (DACs) using A direct aspiration first-pass technique (ADAPT) is equally effective and safe. $^{2}$ However, both techniques (ie, aspiration and stent retrievers) are often used together. ${ }^{3}$ Most interesting, even in the COMPASS trial, stent retrievers were used in $21 \%$ of patients in the aspirationfirst group and DACs were used in most of the stent retriever firstline group. ${ }^{2}$ For all practical purposes, a DAC used in stroke thrombectomy is expected to be compatible for use with stent retrievers. However, we encountered 2 separate cases of unexpected damage to JET 7 Reperfusion Catheters with XTRA FLEX technology (Penumbra) when they were used in conjunction with a stent retriever. Below are the brief reports of the 2 cases.

\section{Incident 1}

An 82-year-old woman presented with acute left hemiparesis and an NIHSS score of 20. Her right ICA was occluded from the distal cervical segment to the ICA termination. Intravenous rtPA was

Received June 4, 2020; accepted after revision July 21.

From the Department of Neuroradiology (J.P.T.), National Neurosciences Institute, Singapore; Division of Neurology (L.Y.) and Division of Interventional Radiology (G.A.), Department of Diagnostic Imaging, National University Hospital, Singapore; and Departments of Medicine (L.Y.) and Radiology (G.A.), Yong Loo Lin School of Medicine, National University of Singapore, Singapore.

Please address correspondence to Anil Gopinathan, MBBS, MD, FRCR (UK), FAMS, Division of Interventional Radiology, National University Hospital, 5 Lower Kent Ridge Rd, 119074 Singapore; e-mail: ivyanil10@gmail.com

Indicates article with supplemental on-line photos.

http://dx.doi.org/10.3174/ajnr.A6804 administered, and the patient was taken for mechanical thrombectomy under sedation and local anesthesia. An angiogram performed with a Neuron MAX 6F 088 (Penumbra) long sheath in the distal common carotid artery showed no antegrade flow beyond the mid-cervical ICA (On-line Fig 1). Aspiration thrombectomy was attempted using a JET 7 catheter introduced through the Neuron MAX over a Velocity delivery microcatheter (Penumbra) and a Transend 0.014-inch microwire (Boston Scientific). After 2 passes and extraction of a large volume of thrombus, the petrous and cavernous parts of the ICA were now patent, but the supraclinoid ICA was still occluded (On-line Fig 2). At this stage, to address excessive patient movement, sedation was converted to general anesthesia. Next, the distal ICA occlusion was crossed with the Velocity microcatheter, and a $4 \times 20 \mathrm{~mm}$ Trevo ProVue (Stryker) stent retriever was deployed from the right M1 into the supraclinoid ICA. The microcatheter was gently removed, while allowing the JET 7 to close over the proximal few millimeters of the stent retriever with continuous aspiration (using the ENGINE aspiration pump [Penumbra]) until there was no back flow. After 3 minutes of stent dwell time, the whole assembly (ie, the stent retriever partially corked at the distal end of the aspiration catheter and the aspiration catheter itself) was withdrawn with continuous suction on the JET 7 as well as the Neuron MAX (On-line Fig 3). Two passes with this technique cleared most of the clots from the supraclinoid ICA with a small residual clot at the proximal M1-ICA termination.

At this stage, while preparing the aspiration catheter, we noted that its distal end, approximately $15 \mathrm{~mm}$ proximal to its tip, was frayed. On connecting the catheter to the pressurized saline flush, the tip of the JET 7 started to balloon (Fig 1). This damaged JET 7 was replaced with an ACE68 aspiration catheter (Penumbra), and by means of the same technique, the procedure was completed to achieve TICI 3 reperfusion (On-line Fig 4). The total 


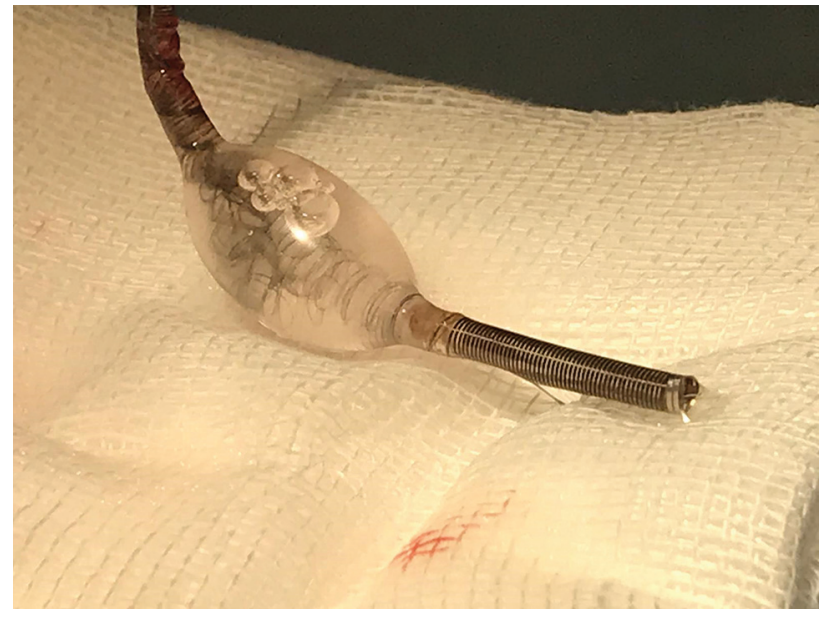

FIG 1. The JET 7 catheter used in the thrombectomy for patient 1 shows ballooning of its distal end with separation of the reinforcing braids from the surrounding polymer.

procedure time was 50 minutes, and the time from ictus to recanalization was 7 hours. A postprocedural CT scan on day 4 did not show any hemorrhagic changes or new infarcts. However, there was no significant clinical improvement in the neurologic deficits at the 3-month follow-up with a modified Rankin Score (mRS) of 4, starting from a baseline of 3 .

\section{Incident 2}

An 88-year-old woman presented with right M1 MCA occlusion and an NIHSS score of 12. Because she was outside the time window for intravenous thrombolysis, she was directly transferred to the angiosuite for mechanical thrombectomy. With the intention of using the direct aspiration first-pass technique, a JET 7 catheter was advanced over a 3MAX (Penumbra) and Transend 0.014inch microcatheter-wire assembly, through a $6 \mathrm{~F}$ Neuron MAX. However, it could not be navigated past the ledge of the ophthalmic artery origin. Hence, the procedure was converted to stent retriever thrombectomy. A $6 \times 30 \mathrm{~mm}$ Solitaire- 2 stent retriever (Medtronic) was deployed across the occlusion and allowed to integrate with the clot for 4 minutes. The Solitaire and the microcatheter were retrieved through the JET 7 with continuous suction using the ENGINE aspiration system. While we withdrew the stent retriever, the JET 7 spontaneously advanced beyond the ophthalmic segment of the ICA and wedged at the right M1-clot interface. Therefore, the operator decided to completely retrieve the Solitaire-microcatheter combination and left the JET 7 catheter in situ. The aspiration was continued for a further 3 minutes during which the aspiration tubing demonstrated slow continuous movement of blood toward the canister before removal of the JET 7. A subsequent angiogram showed TICI 3 reperfusion. When the JET 7 was flushed to check for clots ex vivo, we noted a similar ballooning (Fig 2) of the distal aspect of the catheter as described in case 1 .

\section{DISCUSSION}

In this report, we have described 2 instances of unforeseen damage to the JET 7 reperfusion catheter encountered during

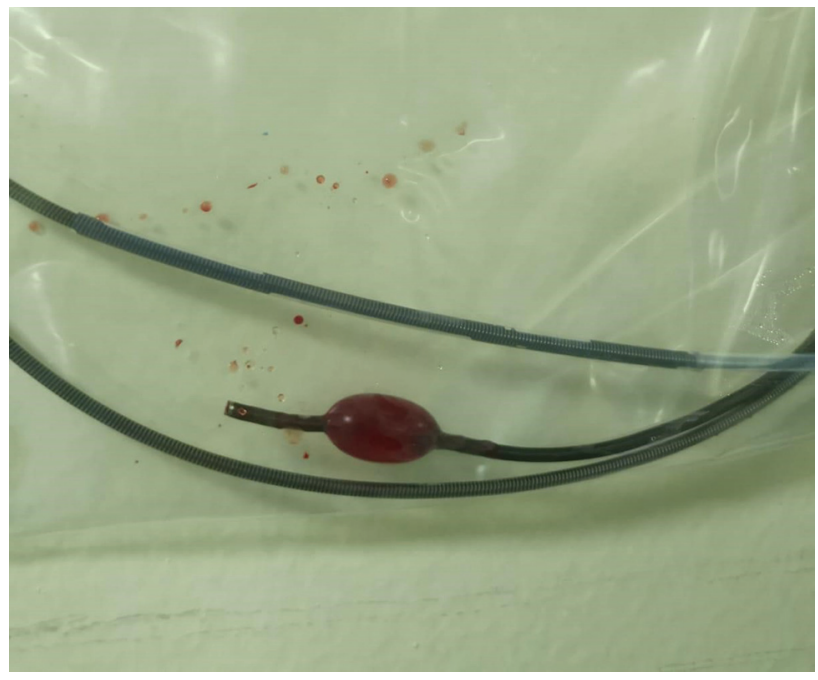

FIG 2. The damaged distal segment of the JET 7 thrombectomy catheter used in patient 2 has ballooned on flushing.

thrombectomy. These procedures were performed by 2 different operators at different institutions. Between the two, the operators have $>15$ years of experience in neurointervention and have performed $>250$ thrombectomies using various generations of Penumbra aspiration catheters with stent retrievers. The thrombectomy techniques used in these 2 cases are comparable with the steps described in published literature; the first one was like the stent retriever-assisted vacuum-locked extraction technique, and the conventional Solumbra technique was used in the second case. In the Solumbra technique, a stent retriever is placed across the clot, while a DAC is advanced to the clot face. Subsequently, the stent retriever is pulled back into the DAC, while negative pressure within the aspiration catheter is preserved with pump aspiration or a negative-pressure syringe. Meanwhile, in the stent retriever-assisted vacuum-locked extraction technique, the stent retriever is deployed with its proximal segment within the clot and the distal segment beyond it. Next, the DAC under suction is advanced to the clot face with gentle traction on the stent wire until a wedged position with the proximal end of the stent is achieved. This wedged stent retriever-DAC assembly is pulled out as a single unit with suction applied on the guide catheter. ${ }^{4}$ At the time of these incidents, the JET 7 catheter with XTRA FLEX technology had been newly introduced into our practice. The 2 events happened 3 weeks apart. One of authors (G.A.) had used the JET 7 uneventfully with first-pass TICI 3 reperfusion on 3 previous occasions. Similarly, the second operator (J.P.T.) had also used the JET 7 twice for aspiration thrombectomy with good angiographic outcomes and no adverse events.

The exact mechanism of the damage to the catheter in these cases is not clearly understood. Pulling an unsheathed stent through the aspiration catheter could potentially harm its integrity due to the friction between the two. However, in the first case, even this mechanism is not plausible because the stent retriever was pushed out of the distal end of the catheter after removing both the catheter and stent together as a single assembly, ie, without pulling it through the JET 7. We did not encounter similar incidents with use 
of the earlier generations of Penumbra aspiration catheters. However, in the last 5 years, there is an ongoing race to manufacture wider bore aspiration catheters with better flexibility and trackability, without increasing their outer diameters. Intuitively, this pursuit could entail a certain degree of compromise on their strength. Operators need to be cognizant of such trade-offs between the stoutness of a catheter against its flexibility and softness, while choosing them off the shelf. In the 2 cases reported here, the distal end of the catheter, beyond the site of ballooning, could have fragmented and embolized or been retained within the patient. Following this experience, after each pass, we closely examine these aspiration catheters for their integrity before re-introducing them into the cerebral circulation.

Disclosures: Anil Gopinathan-UNRELATED: Consultancy: Stryker, Medtronic, Penumbra*; Payment for Lectures Including Service on Speakers Bureaus: Penumbra, Medtronic, Stryker.* *Money paid to the institution.

\section{Note}

Since this report was submitted, Penumbra has issued a safety alert about this problem. ${ }^{5}$ It states that the safety and efficacy of the use of revascularization devices from other manufactures with JET 7 catheter (XTRA FLEX technology) is unknown. They also advise against injection of contrast media through this catheter.

\section{REFERENCES}

1. Powers W, Rabinstein AA, Ackerson T, et al. Guidelines for the Early Management of Patients with Acute Ischemic Stroke: 2019 Update to the 2018 Guidelines for the Early Management of Acute Ischemic Stroke-A Guideline for Healthcare Professionals from the American Heart Association/American Stroke Association. Stroke 2019;50:e344418 CrossRef Medline

2. Turk AQ, Siddiqui A, Fifi JT, et al. COMPASS trial aspiration thrombectomy versus stent retriever thrombectomy as first-line approach for large vessel occlusion (COMPASS): a multicentre, randomised, open label, blinded outcome, non-inferiority trial. Lancet 2019;393:998-1008 CrossRef Medline

3. Brehm A, Maus V, Tsogkas I, et al. Stent-retriever assisted vacuumlocked extraction (SAVE) versus a Direct Aspiration First Pass Technique (ADAPT) for acute stroke: data from the real-world. $B M C$ Neurol 2019;19:65 CrossRef Medline

4. Maus V, Behme D, Kabbasch C, et al. Maximizing first-pass complete reperfusion with SAVE. Clin Neuroradiol 2018;28:327-38 CrossRef Medline

5. https://www.snisonline.org/wp-content/uploads/2020/08/Penumbra-JET7.pdf 